\title{
Recognizing the sarcoidosis behind the window of infection
}

\author{
Tuğba ILLHAN ${ }^{1}$, Ayten Başak KARAAKIN DINAR ${ }^{1}$, Nizameddin KOCA ${ }^{1}$, Koray AYAR ${ }^{2}$
}

\begin{abstract}
${ }^{1}$ Health Sciences University, Bursa Yüksek İhtisas Training and Research Hospital, Department of Internal Medicine, Bursa, Turkey

${ }^{2}$ Health Sciences University, Bursa Yüksek İhtisas Training and Research Hospital, Department of Internal Medicine , Division of Rheumatology, Bursa, Turkey
\end{abstract}

\begin{abstract}
Sarcoidosis is a chronic multisystemic inflammatory disease of unknown etiology, often associated with pulmonary involvement and characterized histopathologically by noncaseating granulomatous lesions. In this report, we aimed to present a 32-year-old patient who was hospitalized with a pre-diagnosis of cellulite, diagnosed with sarcoidosis based on skin lesions, and whose symptoms completely regressed with sarcoidosis treatment.
\end{abstract}

Turk J Int Med 2021;3(Supplement 1):S87-S89 DOI: $10.46310 /$ tjim. 877255

Keywords: sarcoidosis, cutaneous, erythema nodosum

\section{Introduction}

Sarcoidosis is a chronic multisystemic inflammatory disease of unknown etiology, often associated with pulmonary involvement and characterized histopathologically by noncaseating granulomatous lesions. The most common clinic features are respiratory symptoms (cough, dyspnea, bronchial hyperreactivity), fatigue, night sweat, weight loss, and erythema nodosum. Fifty percent of the sarcoidosis patients are asymptomatic, and their diagnosis are usually made with the detection of the bilateral and/or unilateral, hilar and/or paratracheal fullness, seen in the chest X-ray taken for other reasons. ${ }^{1}$ Cellulitis is a suddenly starting and rapidly spreading infection that affects the skin and subcutaneous adipose tissue. The responsible pathogen is usually Staphylococcus Aureus and Streptococcus Pyogenes. They are generally unilateral lesions in the lower extremities that do not have a sharp boundary with intact skin. It may present with local symptoms such as erythema, swelling, warmth, pain and tenderness or systemic symptoms such as fever, chill, shivering and malaise. These signs of cellulitis sometimes can be confused with erythema nodosum seen in the course of sarcoidosis. ${ }^{2}$

Received:February 9, 2021;Accepted:March 8,2021;Published Online:March 6, 2021 


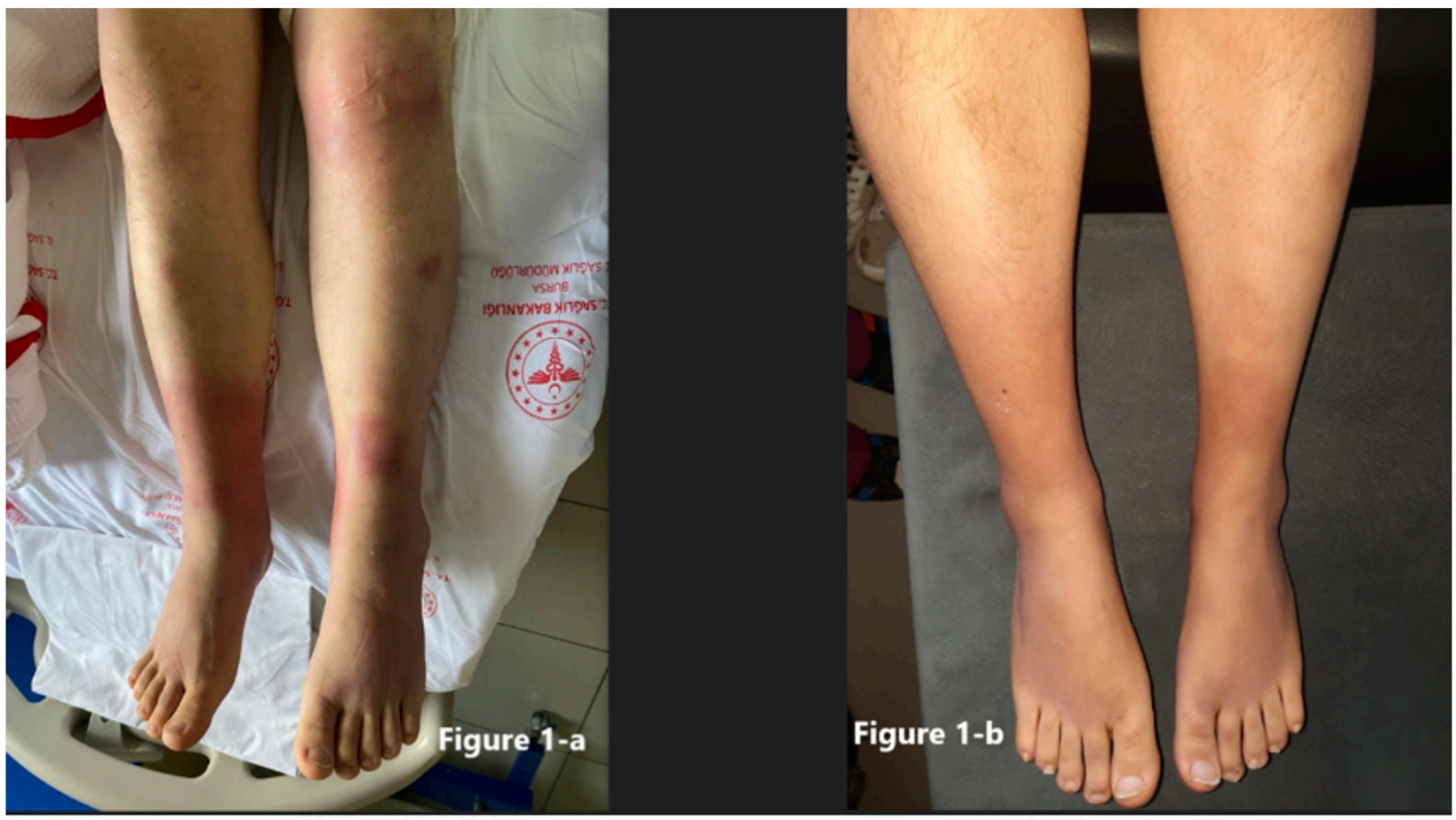

Figure 1. a: skin lesions on admission, b: regression of the skin lesions after treatment.

\section{Case Report}

A 32-year-old patient who have repetitive admission to several hospitals with the complaints of chills, shivering, fever, and redness of the legs in the last month. Various oral and parenteral antibiotics was prescribed with a pre-diagnosis of cellulitis. During the follow-up the patient's complaints did not relieve and the lesion which was initiated from the right tibial region spreaded to both lower and upper extremities (Figure 1a). The lower extremity superficial tissue ultrasonography was reported as cellulitis. He was hospitalized with the pre-diagnosis of multiple cellulitis for further examination. The history of the patient related to the lesions reveals that the lesion was first appeared three weeks ago and progressed despite the treatments given in the outpatient clinic. Clinical and laboratory findings did not improve despite antibiotic treatment. Erythema nodosum was considered as a differential diagnosis. Chest X-ray reveals bilateral hilar lymphadenopathy. Steroid and indomethacin treatment was initiated with the pre-diagnosis of sarcoidosis, and antibiotherapy was stopped. During follow-up, the skin lesions were completely resolved within 3 days (Figure 1b). Further evaluation for the other organ involvement of sarcoidosis was performed. No uveitis, cardiac arrythmia, hepatomegaly and splenomegaly were observed. The patient was discharged after the complete clinical and laboratory remission. Keeping the differential diagnoses on a large scale and making the true diagnosis among these differential diagnoses is the key to reach an effective treatment promptly. Although radiological and laboratory tests are powerful guides in diagnosis, the role of history taking, and physical examination is undeniable.

\section{Discussion}

Early diagnosis of the patient with detailed history, physical examination and accurate laboratory and imaging tests are important for starting an effective treatment as soon as possible. The early diagnosis of a systemic, chronic disease affects the prognosis of the disease positively. Early initiation of necessary treatments by early 
recognition of multiple organ involvement and chronic injuries plays an active role in preventing complications related to disease-related chronicity. The most difficult patients are the patient who come with a pre-diagnosis. For this reason, we should approach each patient with an objective perspective and consider possible differential diagnoses, even if they come with a prediagnosis.

\section{Conflict of Interests}

Authors declare that there are none.

\section{Acknowledgment}

This study has been presented in $17^{\text {th }}$ Uludag Internal Medicine National Winter Congress, $6^{\text {th }}$ Bursa Family Medicine Association National Congress, $11^{\text {th }}$ Uludag Internal Medicine Nursing Congress, 5-7 March 2021, Bursa, Turkey.

\section{References}

1. Chao EC, Henry RR. SGLT2 inhibition--a novel strategy for Tekin NS. Sarcoidosis. Turk J Dermatol. 2012;6:80-6 (in Turkish). doi: 10.5152/tdd.2012.18.

2. Mehdi L, Ekinci AP, Baykal C. Retrospective evaluation of risk factors and response in treatment among hospitalized patients with lower-extremity cellulitis. Turk J Dermatol 2016;10:59-64 (in Turkish). doi: 10.4274/tdd.2871. 